\title{
Optimization of soliton ratchets in inhomogeneous sine-Gordon systems
}

\author{
F. G. Mertens, ${ }^{1}$ L. Morales-Molina, ${ }^{2}$ A. R. Bishop, ${ }^{3}$ A. Sánchez, ${ }^{4}$ and P. Müller ${ }^{1}$ \\ ${ }^{1}$ Physikalisches Institut, Universität Bayreuth, D-95440 Bayreuth, Germany \\ ${ }^{2}$ Max-Planck-Institut für Physik komplexer Systeme, Nöthnitzer Strasse 38, D-01187 Dresden, Germany \\ ${ }^{3}$ Theoretical Division and Center for Nonlinear Studies, Los Alamos National Laboratory, Los Alamos, New Mexico 87545, USA \\ ${ }^{4}$ Grupo Interdisciplinar de Sistemas Complejos (GISC), Departamento de Matemáticas, Universidad Carlos III de Madrid, Avenida de la \\ Universidad 30, 28911 Leganés, Madrid, Spain \\ and Instituto de Biocomputación y Física de Sistemas Complejos (BIFI), Universidad de Zaragoza, 50009 Zaragoza, Spain
}

(Received 24 August 2006; published 6 December 2006; publisher error corrected 11 December 2006)

\begin{abstract}
Unidirectional motion of solitons can take place, although the applied force has zero average in time, when the spatial symmetry is broken by introducing a potential $V(x)$, which consists of periodically repeated cells with each cell containing an asymmetric array of strongly localized inhomogeneities at positions $x_{i}$. A collective coordinate approach shows that the positions, heights, and widths of the inhomogeneities (in that order) are the crucial parameters so as to obtain an optimal effective potential $U_{\text {opt }}$ that yields a maximal average soliton velocity. $U_{\text {opt }}$ essentially exhibits two features: double peaks consisting of a positive and a negative peak, and long flat regions between the double peaks. Such a potential can be obtained by choosing inhomogeneities with opposite signs (e.g., microresistors and microshorts in the case of long Josephson junctions) that are positioned close to each other, while the distance between each peak pair is rather large. These results of the collective variable theory are confirmed by full simulations for the inhomogeneous sine-Gordon system.
\end{abstract}

DOI: 10.1103/PhysRevE.74.066602

PACS number(s): 05.45.Yv, 85.25.Cp, 73.40.Ei

\section{INTRODUCTION}

Ratchet or rectification phenomena appear in many different fields ranging from nanodevices to molecular biology [1-5]. In the simplest model a pointlike particle is considered which is driven by deterministic or nonwhite stochastic forces. Under certain conditions related to the breaking of symmetries, unidirectional motion of the particle can take place although the applied force has zero average in time.

These particle ratchets have been generalized to spatially extended nonlinear systems, in which solitons play a similar role to the above point particles [6-12]. In particular, solitons in nonlinear Klein-Gordon systems have been shown to move on the average in one direction, although the driving force has zero time average, if either a temporal or a spatial symmetry is broken.

In the first case, a biharmonic driving force has been used which breaks a time shift symmetry $[13,14]$. Here the mechanism of the ratchet effect has been clarified in detail by a collective variable theory $[15,16]$, which uses the soliton position $X(t)$ and width $l(t)$. Due to the coupling between the translational and internal degrees of freedom, energy is pumped inhomogeneously into the system, generating a directional motion. The breaking of the time shift symmetry gives rise to a resonance mechanism that takes place whenever the width $l(t)$ oscillates with at least one frequency of the external ac force. This ratchet effect has been confirmed by experiments with annular Josephson junctions [17] which can be modeled by sine-Gordon systems; here flux quanta (fluxons) play the role of the solitons. As the external ac force, biharmonic microwaves have been used.

The biharmonic force has recently been replaced by more general periodic forces which can be expressed by Jacobian elliptic functions. There, it turns out that the average soliton velocity exhibits an extremum for a certain value of the modulus of the elliptic functions [18]. This means that there is an optimal form for the periodic force.

Another way to obtain a soliton ratchet is to break the spatial symmetry. This has been demonstrated very recently by the introduction of pointlike inhomogeneities into nonlinear Klein-Gordon systems [19,20]. These inhomogeneities can be modeled by $\delta$ functions, if their spatial extent is much smaller than the characteristic length for the system (the Josephson penetration length in the case of Josephson junctions). In order to achieve a ratchet effect the $\delta$ functions must form asymmetric periodic arrays. This asymmetry translates into an asymmetry of an effective potential $U(X, l)$ which appears in a collective variable theory with the variables $X(t)$ and $l(t)$.

As an example, three $\delta$ functions of equal strength have been positioned in each cell of an array with period $L$. The above theory yielded a nearly perfect agreement with the simulations for the inhomogeneous sine-Gordon system [20]. By choosing different arrangements for the positions of the $\delta$ functions within the cells of the array it was possible to increase the average soliton velocity $\langle v\rangle$ which means that the ratchet system provides better transport.

The aim of this paper is to search for the optimal shape and arrangement of localized inhomogeneities, in the sense that $\langle v\rangle$ becomes as large as possible. This means that instead of $\delta$ functions other functions which represent localized inhomogeneities (e.g., Gaussians, Lorentzians, box-type functions, etc.) have to be tested, and that the arrangement of these inhomogeneities within the cells, but also the length $L$ of the cells in the periodic array, all have to be optimized.

In Sec. II nonlinear Klein-Gordon systems with localized inhomogeneities are introduced. In Sec. III a collective coordinate (CC) theory is developed which results in a set of ordinary differential equations (ODEs) containing an effective potential $U$ which depends on the inhomogeneities. It 
turns out that their most significant features for maximizing $\langle v\rangle$ are their positions, heights, and widths, while their detailed shape is unimportant. For this reason we work with the simplest shape, namely, that of boxes, where $U$ can be calculated analytically. In Sec. IV the optimal effective potential $U_{\text {opt }}$ is obtained by using an ansatz in terms of Jacobi elliptic functions. $U_{o p t}$ essentially exhibits two features: double peaks consisting of a positive and a negative peak with a steep slope in between, and long flat regions between the double peaks. In Sec. V we show that the essential features of $U_{\text {opt }}$ can be produced by choosing appropriately the positions, heights, and widths of box inhomogeneities. In this way very high soliton velocities can be obtained. These results of the CC theory are confirmed by full simulations for the sine-Gordon system with the above inhomogeneities (Sec. VI).

In the last section we stress that an important step in the optimization consists in using inhomogeneities with opposite signs. Both types of inhomogeneities were already used in long Josephson junctions, namely, microresistors (critical current $J_{c}$ decreased) and microshorts $\left(J_{c}\right.$ increased) [21]. However, so far only equidistant arrays of either type were used in experiments. We propose to use a specific combination of both types, resulting in an asymmetric periodic array, which yields very high average soliton velocities both in a collective coordinate theory and in full simulations for the inhomogeneous sine-Gordon system.

Finally, we remark that Abrikosov vortices in Josephson junctions, with axes almost perpendicular to the junction plane, can be regarded as microresistors [22]. Moreover, it is now possible to fabricate $\pi$ Josephson tunnel junctions with a ferromagnetic barrier, which offer several new applications [23]. Another possibility is offered by high- $T_{c}$ superconductors made of granular materials. In this respect, the interplay between the shape and geometry for the grains may lead to a nonsymmetric landscape for the propagation of fluxons [24].

\section{INHOMOGENEOUS NONLINEAR KLEIN-GORDON SYSTEMS}

A ratchet effect has recently been obtained $[19,20]$ by breaking the spatial symmetry of nonlinear Klein-Gordon systems with inhomogeneities introduced via a potential $V(x)$

$$
\phi_{t t}+\beta \phi_{t}-\phi_{x x}+\frac{\partial \widetilde{U}}{\partial \phi}[1+V(x)]=f(t) .
$$

Here $\phi(x, t)$ is a scalar field, $\phi_{x}$ and $\phi_{t}$ are partial derivatives with respect to space and time, $\beta$ is a damping coefficient, $\widetilde{U}(\phi)=1-\cos \phi$ is the sine-Gordon (SG) potential, and $\widetilde{U}=\frac{1}{4}\left(\phi^{2}-1\right)^{2}$ the $\phi^{4}$ potential. As both cases have produced very similar results [20], we will concentrate on the SG model in the following, but we will always indicate which results also hold for the $\phi^{4}$ model. $f(t)=A \sin \left(\omega t+\delta_{0}\right)$ is an external ac force with amplitude $A$, frequency $\omega$, and initial phase $\delta_{0}$.

$V(x)$ consists of periodically repeated cells of length $L$; each cell $n$ contains an asymmetric array of strongly local- ized inhomogeneities $g_{i}(x)$ which are placed at positions $x_{i}$ within the cell; i.e.,

$$
V(x)=\sum_{n} \sum_{i} g_{i}\left(x-x_{i}-n L\right) .
$$

So far only the case of pointlike inhomogeneities was considered, which can be modeled by $\delta$ functions. Taking three $\delta$ functions with equal strengths and choosing their positions $x_{i}$ in certain asymmetric ways, a sine-Gordon soliton (also named a kink in the following) moves either to the right or to the left on the average [19]. Depending on the choice of the driving frequency $\omega$, qualitatively different results were obtained for the modulus $\bar{v}$ of the average soliton speed $\langle v\rangle$ as a function of the driving amplitude $A$ : (a) For the low frequency $\omega=0.015$ there is an up and down staircase with the maximum average velocity $v_{\max }=0.03820$, (b) for the intermediate frequency $\omega=0.05$ there are five "windows" (regions of $A$ with constant $\bar{v}>0$ ) with $v_{\max }$ $=0.03183$, and (c) for the relatively high frequency $\omega=0.1$ there is only one window with height $v_{\max }=0.063666$. For even higher frequencies, $\bar{v}$ vanishes for all amplitudes $A$. The results for $\phi^{4}$ nonlinearity are quite similar.

$v_{\max }$ is always small compared to the critical velocity $c$ which is unity for the dimensionless system (1). For long Josephson junctions $c$ is the Swihart velocity [25].

The aim of this paper is to optimize both the shape and the array of the inhomogeneities in the sense that $\bar{v}$ becomes as large as possible. Naturally, there are still other criteria for the optimization. For example, one can try to maximize the area under the curve $\bar{v}$ vs amplitude $A$; this will be discussed later. Other optimization strategies include studying the energy efficiency. We do not consider this approach here, but we refer the reader to Ref. [26].

In order to achieve any kind of optimization, it is necessary to have a theory which allows calculation of $\langle v\rangle$ for a given array of inhomogeneities. A CC theory with two variables (2-CC), namely, position $X(t)$ and width $l(t)$ of the soliton, has turned out to be very successful [19], in the sense that in the case of $\delta$ functions the results for $\langle v\rangle$ as a function of the driving amplitude $A$ agree very well with the simulations for the original system [full numerical solution of Eq. (1)]. Deviations between theory and simulation are found only in the case of strong driving $[A=O(1)]$. For even stronger driving the theory is no longer applicable, because in the simulations kink-antikink pairs appear spontaneously and scatter with the soliton under consideration. The 2-CC theory is characterized by two ODEs for $X(t)$ and $l(t)$, which contain forces that are defined as partial derivatives of an effective potential $U(X, l)$, which is calculated and discussed in the next section.

We take for our computations a rather large damping, choosing $\beta=1$, for the following reasons.

(a) Our CC theory does not take into account the phonons which are radiated by the soliton due to its acceleration by the driving force. These phonons are quickly damped out when the damping is large.

(b) We want to avoid several phenomena which can occur for very small damping, e.g., chaotic behavior and current reversals. 
Finally, we remark that the spatial symmetry can be broken also by the introduction of an additive inhomogeneity $\gamma(x)$ in Eq. (1), instead of our multiplicative inhomogeneity $(\partial \tilde{U} / \partial \phi) V(x)$. In the case of long Josephson junctions an additive inhomogeneity has been experimentally realized by a current injection with a profile $\gamma(x)[27,28]$. This produces an effective potential which is similar to an asymmetric sawtooth potential [28].

\section{COLLECTIVE COORDINATE THEORY}

For the unperturbed sine-Gordon equation, i.e., Eq. (1) without damping, inhomogeneity, and driving, the onesoliton solution reads

$$
\phi_{0}(x, t)=4 \arctan \left[\exp \left(\gamma \frac{x-v t}{l_{0}}\right)\right],
$$

where $v$ is the soliton velocity, $l_{0}=1$ is the rest width, and $l_{0} / \gamma=l_{0} \sqrt{1-v^{2}}$ is the Lorentz-contracted width.

For perturbed nonlinear Klein-Gordon systems the socalled Rice ansatz [29] turned out to be very successful. For example, for the ac-driven $\phi^{4}$ system an unexpected resonance was predicted (and confirmed by simulations), which is situated in the gap below the phonon spectrum at half the frequency of the internal mode of the $\phi^{4}$ kink $[30,31]$. The Rice ansatz for SG systems reads

$$
\phi(x, X, l)=4 \arctan \left[\exp \left(\frac{x-X(t)}{l(t)}\right)\right]
$$

with soliton position $X(t)$ and width $l(t)$. The 2-CC theory for the perturbed SG equation (1) yielded a set of two coupled ODEs [20]

$$
\begin{gathered}
M_{0} l_{0} \frac{\ddot{X}}{l}+\beta M_{0} l_{0} \frac{\dot{X}}{l}-M_{0} l_{0} \frac{\dot{X} i}{l^{2}}=F^{a c}+F^{i n h}, \\
\alpha M_{0} l_{0} \frac{\ddot{l}}{l}+\beta \alpha M_{0} l_{0} \frac{\dot{l}}{l}+M_{0} l_{0} \frac{\dot{X}^{2}}{l^{2}}=K^{i n t}+K^{i n h},
\end{gathered}
$$

where $\alpha=\pi^{2} / 12, M_{0}=8, l_{0}=1$, and

$$
F^{a c}=\int_{-\infty}^{\infty} d x f(t) \frac{\partial \phi}{\partial X}=-q f(t)
$$

with the topological charge $q=2 \pi$.

$$
K^{\text {int }}=-\frac{\partial E}{\partial l}
$$

is a force that arises from the soliton energy

$$
E(\dot{X}, l, \dot{l})=\frac{1}{2} \frac{l_{0}}{l} M_{0} \dot{X}^{2}+\frac{1}{2} \frac{l_{0}}{l} \alpha M_{0} \dot{l}^{2}+\frac{1}{2} M_{0}\left(\frac{l_{0}}{l}+\frac{l}{l_{0}}\right),
$$

including internal energy due to width oscillations.

There are two forces that appear due to the potential $V(x)$ in Eq. (1), viz.,

$$
\begin{aligned}
& F^{i n h}=-\int_{-\infty}^{\infty} d x \frac{\partial \tilde{U}}{\partial \phi} \frac{\partial \phi}{\partial X} V(x)=-\int_{-\infty}^{\infty} d x \frac{\partial \widetilde{U}}{\partial X} V(x):=-\frac{\partial U}{\partial X}, \\
& K^{i n h}=-\int_{-\infty}^{\infty} d x \frac{\partial \tilde{U}}{\partial \phi} \frac{\partial \phi}{\partial l} V(x)=-\int_{-\infty}^{\infty} d x \frac{\partial \widetilde{U}}{\partial l} V(x):=-\frac{\partial U}{\partial l} .
\end{aligned}
$$

In this way the effective potential

$$
U(X, l)=\int_{-\infty}^{\infty} d x \widetilde{U}(\phi) V(x)
$$

is introduced that characterizes the influence of the inhomogeneities on the soliton dynamics. We remark that all the above results also hold for the $\phi^{4}$ model: In this case $\phi$ $=\tanh [(x-X) / l]$ and the parameters are $q=2, M_{0}=2 \sqrt{2} / 3$, $l_{0}=\sqrt{2}$, and $\alpha=\left(\pi^{2}-6\right) / 12$.

Before we specify $V(x)$ we evaluate $\tilde{U}(\phi)$. First we derive the relation $\tilde{U}\left(\phi_{0}\right)=\left(\partial \phi_{0} / \partial z\right)^{2} /\left(2 \gamma^{2}\right)=2 \operatorname{sech}^{2}(\gamma z)$, with $z=x$ $-v t$, by inserting $\phi_{0}$ into the unperturbed SG equation. However, $\widetilde{U}(\phi)$ cannot be obtained in this way because $\phi(x, X, l)$ is not an exact solution, but rather an ansatz. However, we can generalize the above relation for $\widetilde{U}\left(\phi_{0}\right)$ to $\widetilde{U}(\phi)=\frac{1}{2} l^{2} \phi_{z}^{2}$ $=2 \operatorname{sech}^{2}(z / l)$, with $z=x-X$, and this can be verified by, e.g., MATHEMATICA [32].

Thus we finally obtain

$$
U(X, l)=\int_{-\infty}^{\infty} d x 2 \operatorname{sech}^{2} \frac{x-X}{l} V(x) .
$$

Now we can insert the superposition (2) of localized inhomogeneities $g_{i}$ and obtain

$$
\begin{gathered}
U(X, l)=\sum_{n} \sum_{i} U_{i}^{(n)}, \\
U_{i}^{(n)}=\int_{-\infty}^{\infty} d x 2 \operatorname{sech}^{2} \frac{x-X}{l} g_{i}\left(x-x_{i}-n L\right) .
\end{gathered}
$$

We can check that the effective potential is indeed periodic, $U(X+L, l)=U(X, l)$, because the sum goes over an infinite number of unit cells $n$ of length $L$.

Our next step is to evaluate $U_{i}^{(n)}$ for various strongly localized functions $g_{i}$ and to discuss which are the most important features of the inhomogeneities. We have tested Gaussians, Lorentzians, and box functions and it turns out that there are only two important features of $g_{i}$, namely, the height and width. For the above three cases, the $U_{i}^{(n)}$ are always bell shaped, and the desired asymmetry of $U$ depends most strongly on the relative positions of the $U_{i}^{(n)}$ within the cells. In other words, the most important features of the inhomogeneities $g_{i}$ are their positions $x_{i}$, then come their heights and widths, while their detailed shape is unimportant.

For this reason, we can choose the simplest case, namely, the box functions, which has the additional advantage that $U(X, l)$ can be calculated analytically (in contrast to the other 
two cases). The box function $g_{i}$ at $x_{i}$ in cell $n=0$ is defined as

$$
g_{i}= \begin{cases}h_{i} & \text { for } x_{i}-b_{i} \leqslant x \leqslant x_{i}+b_{i}, \\ 0 & \text { otherwise }\end{cases}
$$

This yields

$$
U_{i}^{(o)}=2 h_{i} l \sinh \frac{2 b_{i}}{l} \operatorname{sech} \frac{X-x_{i}+b_{i}}{l} \operatorname{sech} \frac{X-x_{i}-b_{i}}{l},
$$

and we can check that in the limit $h_{i} \rightarrow \infty, b_{i} \rightarrow 0$ with finite $\epsilon_{i}:=2 b_{i} h_{i}$ the result for a $\delta$-function inhomogeneity [19] can be regained: $U_{i}^{(0)}=2 \epsilon_{i} \operatorname{sech}^{2}\left[\left(X-x_{i}\right) / l\right]$. The final result for the effective potential (14) is

$$
U(X, l)=\sum_{n} \sum_{i} 2 h_{i} l \sinh \frac{2 b_{i}}{l} \operatorname{sech} \frac{Z_{+}}{l} \operatorname{sech} \frac{Z_{-}}{l}
$$

with $Z_{ \pm}=X-x_{i}-n L \pm b_{i}$

In this way the optimization problem is solved, but only in principle. We choose a set of box inhomogeneities at the positions $x_{i}$ within the cells, numerically solve the ODEs (5) and (6) for $X(t)$ and $l(t)$, and compute the average soliton velocity, defined by

$$
\langle v\rangle=\langle\dot{X}\rangle=\lim _{t \rightarrow \infty} \frac{\langle X(t)-X(0)\rangle}{t},
$$

choosing a sufficiently long integration time. Then the procedure has to be repeated for many values of the driving amplitude $A$ (keeping the driving frequency $\omega$ fixed), in order to find the maximal soliton speed

$$
v_{\max }=\max \{|\langle v\rangle|\}
$$

for that set of parameters. Finally, we would like to obtain $v_{\max }$ for a low, a medium, and a relatively high frequency, because it is known from the case of the $\delta$-function inhomogeneities that the curves $\langle v\rangle$ vs $A$ differ qualitatively for the above choice of frequencies (see Sec. II).

In practice the whole procedure cannot work because the dimension of the parameter space is far too high. For example, if one takes three inhomogeneities per cell, as in the case of the $\delta$ functions, one has ten parameters, namely, three positions, heights, and widths of the boxes, plus the cell length $L$. As one has to make sweeps in $A$ in order to get $v_{\max }$ for every parameter set, it is practically impossible to explore the ten-dimensional parameter space. Obviously, we have to find a more efficient way to obtain an optimal set of inhomogeneities.

\section{OPTIMAL EFFECTIVE POTENTIAL}

Our approach is not to work directly with the potential $V(x)$ in the inhomogeneous SG equation (1), but to design first an optimal effective potential $U_{o p t}$ for a given driving force, i.e., for fixed $A$ and $\omega$. When $U_{\text {opt }}$ has been obtained, it can be represented (approximately) by the superposition (18) of the pulse-shaped contributions $U_{i}^{(n)}$. That is, the many parameters in Eq. (18) could be determined for instance by a least-squares fit of $U$ to $U_{o p t}$, which is much more efficient than a search in the high-dimensional parameter space.

For simplicity we will not try to find $U_{o p t}(X, l)$ within the 2-CC approach. It will turn out to be sufficient to find an optimal potential within the 1-CC theory which starts with the ansatz

$$
\phi_{0}(x, X)=4 \arctan \{\exp [\gamma(x-X(t)))]
$$

with

$$
\gamma=1 / \sqrt{1-\dot{X}^{2}} .
$$

This yields one ODE [20]

$$
\gamma^{3} M_{o} \ddot{X}+\gamma \beta \dot{X}=F^{a c}+F^{i n h},
$$

with $F^{i n h}=-\partial U / \partial X$ and

$$
U=\int_{-\infty}^{\infty} d x \tilde{U}(\phi) V(x)=\int_{-\infty}^{\infty} d x 2 \operatorname{sech}^{2}[\gamma(x-X)] V(x) .
$$

Here, $\gamma^{3} M_{0}$ is the so-called longitudinal relativistic mass. So far only the nonrelativistic limit $(\gamma=1)$ of Eq. (23) was considered in the context of the $\delta$-function inhomogeneities $[19,20]$. Here we need the relativistic version, because our optimization will yield velocities that are no longer small compared to the critical velocity $c=1$.

As noted above, we do not consider here the expression (24) for $U$ in terms of $V(x)$, but we want to find quite generally the optimal $U$ for a given driving force $F^{a c}$. $U_{\text {opt }}$ must be asymmetric and periodic. As the most general periodic functions are the Jacobi elliptic functions, we use the ansatz

$$
U_{\text {opt }}(X)=-\epsilon \operatorname{sn}(k X, m) \operatorname{cn}(k X, m),
$$

where $m$ is the modulus of the elliptic functions, $k$ $=2 K(\mathrm{~m}) / L$ is a generalized wave number, and $K(\mathrm{~m})$ is the complete elliptic integral of the first kind.

This type of ansatz was first introduced in [18] as a generalized driving force to break the temporal symmetry in ratchet systems, namely, $f(t) \equiv F_{\text {ellip }}(t)=\epsilon \operatorname{sn}(\Omega t, m)$ $\operatorname{cn}(\Omega t, m)$ [where $\Omega=2 K(m) / T$ with period $T$ ]. Using only symmetry arguments, and taking into account the first two terms of the Fourier series of $F_{\text {ellip }}(t)$, it was shown in [18] that the optimal value for $m$ is 0.960057 , independent of the details of the ratchet models. However, in our case we will show that the situation is more complicated when such an ansatz is taken for the potential $U(X)$ in the particle ratchet (23).

The negative sign in front of $\epsilon>0$ in (25) is chosen in order to achieve a positive $\langle v\rangle$, i.e., average motion to the right. The specific combination (25) of elliptic functions is chosen because there is no ratchet effect in two limiting cases: For $m=0$ the effective potential is sinusoidal, and for $m=1$ it is zero (except for a set of points with Lebesgue measure zero). Thus we can expect that an optimal value $m_{\text {opt }}$ naturally exists in the interval $0<m<1$, under the condition that there is any ratchet effect for the chosen set of parameters $\epsilon, L$ in $U(X)$ and $A, \omega$ in the driving force. 


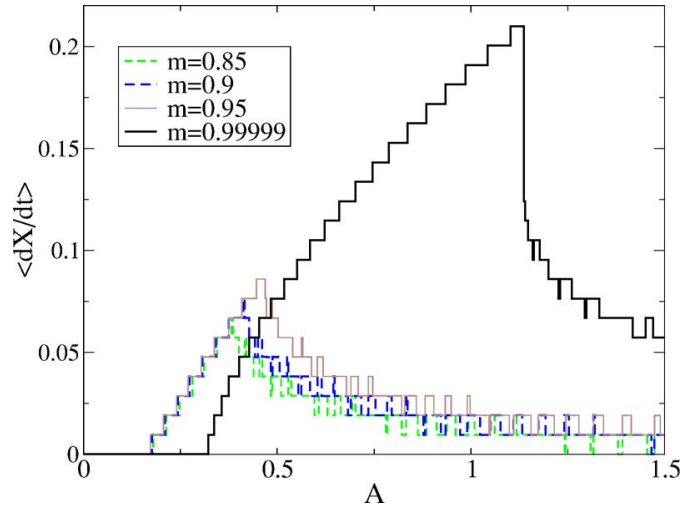

FIG. 1. (Color online) Mean soliton velocity vs amplitude $A$ of the ac force for different moduli $m$ of the elliptic functions in the ansatz (25) for the effective potential. The parameters used are $L$ $=4, \beta=1, \omega=0.015$, and $\epsilon=-2$.

Our problem now is to find the optimal modulus $m$, i.e., the optimal shape of the potential $U(X)$ in the ODE (23), which formally is a relativistic particle ratchet model. In Fig. $1\langle v\rangle$ vs $A$ is plotted for various values of $m$ and we can see that the maximal speed $v_{\max }$ gradually increases with increasing $m$. However, when $m$ is close to 1 , there is a dramatic increase of $v_{\max }$. The reason for this can be discussed by considering the change of shape of $U(X)$ in Fig. 2: When $m$ is increased (but is not yet close to 1 ) the negative slopes in $U(X)$ become steeper and the positive slopes become weaker. Both together increases the asymmetry of the potential; thus the symmetry breaking is augmented, which explains why $v_{\max }$ grows. In fact, $U(X)$ looks like a (smoothed) sawtooth potential that has widely been used in the literature.

However, when $m$ becomes close to 1 , a new feature appears in $U(X)$, namely, long flat regions. Their lengths increase as $m$ gets even closer to 1 , so eventually $U(X)$ essentially exhibits two features: (1) double peaks, each consisting of a positive and a negative peak with a steep slope in between, and (2) long flat regions between the double peaks.

A potential with similar features was proposed for a ratchet system driven by a multiplicative white noise to enhance the coherent transport [33].

Before we proceed with our goal, namely, to find the optimal inhomogeneities which produce the above features, we

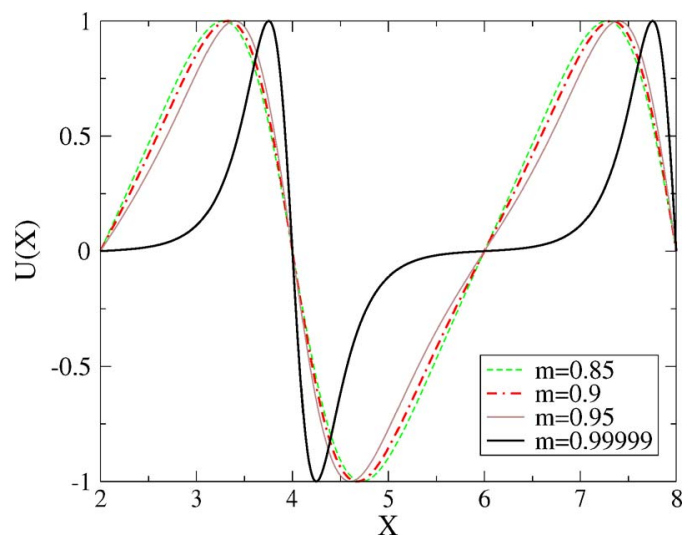

FIG. 2. (Color online) Effective potential (25) for different values of the modulus $m$ of the elliptic functions; $L=4, \epsilon=-2$.

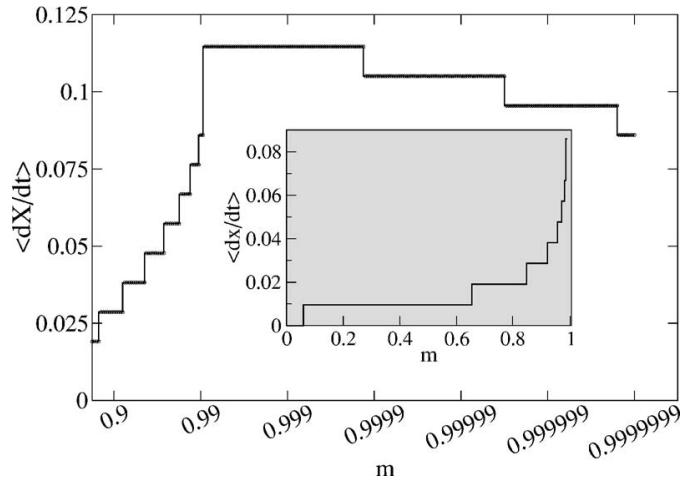

FIG. 3. Mean soliton velocity vs the modulus $m$ of the elliptic functions in the ansatz (25) for the effective potential; $L=4, A$ $=0.6, \beta=1, \omega=0.015, \epsilon=-2$.

want to discuss the staircase structure of $\langle v\rangle$ vs $A$ in Fig. 1. This structure is described by

$$
\langle v\rangle=\frac{i}{j} \frac{L}{T}=\frac{i}{j} v_{\text {step }}, \quad i, j \text { integer. }
$$

The step height $v_{\text {step }}=L / T$ does not depend on the shape, but only on the period of $U(X)$, and on the period $T=2 \pi / \omega$ of the ac force.

Formula (26) should hold quite generally according to most of the literature ([4] and references therein). However, Eq. (26) was explicitly calculated only for a special case, namely, an asymmetric sawtooth potential and a piecewise constant driving force [34]. Our results confirm Eq. (26), and so do the results for the effective potential stemming from $\delta$-function inhomogeneities [19]. Moreover, we observe that $\langle v\rangle$ changes in steps also as a function of other parameters. For the following it will be important that $\langle v\rangle$ as a function of $m$ first increases, then decreases in steps (Fig. 3). This means that $v_{\max }$ is reached for a very narrow range of $m$, which we denote by $\left[m_{\text {opt }}\right]$. The larger $A$, the closer $\left[m_{\text {opt }}\right]$ is to 1 . For a larger value of the frequency, $\omega=0.05$, the same effect shows up, namely, it is found that the ratchet transport is enhanced for $m$ very close to 1 provided that $A$ is not too small.

\section{OPTIMAL INHOMOGENEITIES}

Our approach was to construct an array of inhomogeneities which produces an effective potential whose shape is approximately equivalent to the shape of $U_{o p t}$, i.e., it must exhibit the two essential features found in $U_{o p t}$ in the previous section. The required effective potential can be obtained in four steps [here we skip working with $U(X)$ from the 1-CC theory and proceed directly with $U(X, l)$ in Eq. (18)].

(1) A single box inhomogeneity produces the single peak (17); two boxes with equal width $\left(b_{1}=b_{2}=b\right)$ and equal height, but opposite signs $\left(h_{1}=h, h_{2}=-h\right)$, produce the desired double-peak structure, if they are placed at positions $x_{1}, x_{2}$ not too far separated.

(2) As to the second feature of $U_{\text {opt }}$, the long flat regions between the double peaks can easily be obtained by choosing 
a large period $L$, compared to the width of the double peaks. For the widths discussed below, a good range is $L=4-8$; here $\langle v\rangle$ depends only weakly on $L$.

(3) We need to make the negative slope within a double peak very steep, and the positive slope between double peaks very weak, because these are features of $U_{o p t}$. Both features can be achieved by making the two boxes narrower and higher (keeping the area fixed). Unfortunately, the effect of this procedure is limited by the fact that the two peaks $U_{1}^{(0)}$ and $U_{2}^{(0)}$, which are produced by the two boxes, have a minimum width of about 1 [for $l \simeq O(1)$ ] [see Eq. (17)]. This width is practically the same as that of the $\operatorname{sech}^{2}$ peak produced by a $\delta$ function inhomogeneity [see below Eq. (17)].

(4) Because of the limitations of step 3, we position the two boxes closer together. This would make the negative slope steeper, if there were not a partial compensation of the positive and negative contributions $U_{1}^{(0)}$ and $U_{2}^{(0)}$ which reduces the heights of the two peaks. One can compensate this effect by further increasing the height $h$ of the boxes and thus the peak heights. The optimal double-peak structure is produced by two very narrow boxes which are as close together as possible, namely, touching each other without overlapping (e.g., $\left.x_{1}=0.8, x_{2}=1.0, b=0.1\right)$. Then the height can be increased as long as $v_{\max }$ is reached for $A \leqslant 1$ (for the above example $h=25$, see Fig. 4). There is no value in increasing the amplitude of the driving force beyond $A \simeq 1$ because in the simulations for the original partial differential equation (1) kink-antikink pairs may then appear spontaneously and degrade the ratchet effect (see also the end of Sec. II).

The above four steps together yield an enormous gain for $v_{\max }$ in the order of $300 \%$. In Fig. 4(a) the maximal value of $\langle v\rangle$ is 0.1528 for $\omega=0.015$, which has to be compared with the best value 0.0382 for the case of three $\delta$-function inhomogeneities with the same $\omega$ [19]. The value 0.1528 for $v_{\max }$ is indeed quite high, taking into account that this is an average over positive and negative velocities [cf. Eq. (19)]. Even larger values of $v_{\max }$ can be obtained by choosing a small damping parameter, but we do not consider this regime for the reasons given at the end of Sec. II.

The above pair of very narrow and very high boxes that touch each other produces an effective potential which can be approximated very well by that resulting from a $\delta^{\prime}$-function inhomogeneity. This can be shown by considering in Eq. (18) the limit $b \rightarrow 0, h \rightarrow \infty$ with finite $\epsilon:=(2 b)^{2} h$ for a box pair at $x_{1}$ and $x_{2}=x_{1}+2 b$ in cell $n=0$. The limit yields

$$
U^{(0)}(X, l)=-\frac{4 \epsilon}{l} \tanh \frac{X-x_{1}}{l} \operatorname{sech}^{2} \frac{X-x_{1}}{l}
$$

which is identical with $U^{(0)}$ stemming from a $\delta^{\prime}$-function inhomogeneity $g_{1}=\epsilon \delta^{\prime}\left(x-x_{1}\right)$.

\section{COMPARISON WITH SIMULATIONS}

In order to check the predictions of the above collective coordinate approach with the optimized effective potential constructed in the previous section, we have performed simulations for the full sine-Gordon system (1) with the box
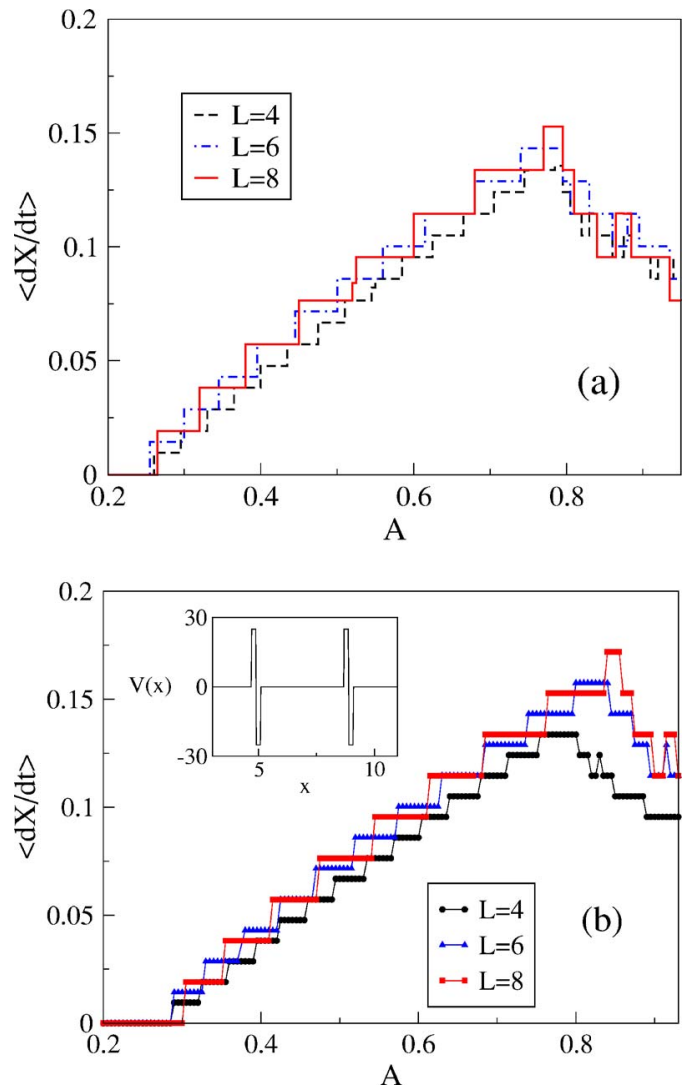

FIG. 4. (Color online) Mean velocity vs amplitude $A$ of the ac force. (a) Numerical solution of the CC equations (5)-(11) with $U(X, l)$ given by Eq. (18). (b) Simulations of Eq. (1) with the expression for $V(x)$ given by Eqs. (2) and (16). $V(x)$ is shown in the inset for $L=4$. The parameters used are $\beta=1, \omega=0.015, h=25, b$ $=0.1, x_{1}=0.8, x_{2}=1.0$.

inhomogeneities that produce the above effective potential: two narrow and high boxes per cell that have opposite signs and touch each other. We have numerically solved Eq. (1) using the Heun scheme [35] which yields the same results as the Strauss-Vázquez scheme [36], but considerably faster; another advantage of the Heun scheme is the possibility to include thermal noise [20]. The spatial and temporal integration steps were $\Delta x=0.025$ and $\Delta t=0.005$. The spatial interval for the simulations had a length of 190 units. The simulations were performed taking as initial condition a kink soliton Eq. (4) at rest, which we allow to evolve. Then, after some transient time, we compute the mean velocity integrating over a period of time. This process is repeated again by varying the amplitude of the force, thus sweeping over the whole interval for the force amplitude. We have checked that the computation of the mean velocity does not change over several periods.

The results in Fig. 4(b) show a very good qualitative agreement with the CC approach results in Fig. 4(a). Interestingly the simulation results are more sensitive to the choice of the cell length than the CC approach results: both the maximum $v_{\max }$ of the average velocity and its position change more when $L$ is increased from 4 to 8 . The best value is $v_{\max }=0.172$, which is $350 \%$ higher than the best result 0.0382 for the $\delta$ function inhomogeneities [19]. In this case 

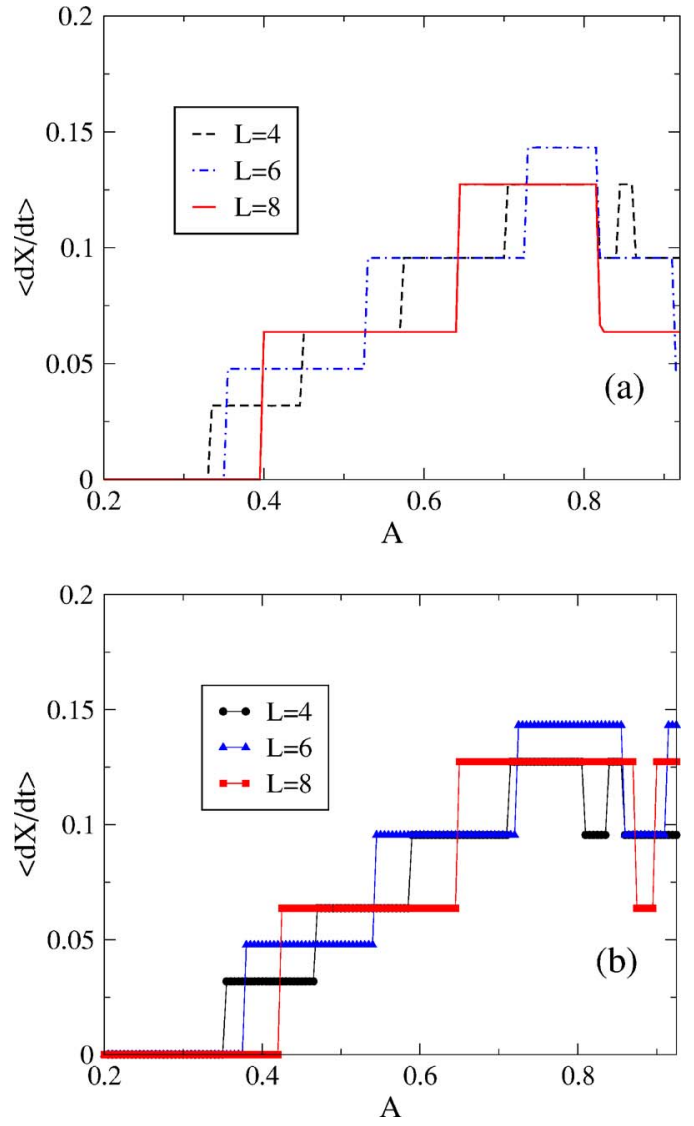

FIG. 5. (Color online) Mean velocity vs amplitude $A$ of the ac force. (a) Numerical solution of the CC equations (5)-(11). (b) Simulations of Eq. (1) with the expression for $V(x)$ given by Eqs. (2) and (16). The parameters used are $\beta=1, \omega=0.05, b=0.1, h$ $=25, x_{1}=0.8, x_{2}=1.0$.

the highest value of the soliton speed $|v(t)|$ is about 0.5 which has to be compared with the critical velocity $c=1$.

So far we have only considered the case of a low frequency $(\omega=0.015)$ of the driving force. Here both the $\delta$ function and the box inhomogeneities produce a staircase in $\langle v\rangle$ vs $A$. However, for higher frequencies the situation changes: For $\omega=0.05$ the boxes still yield a staircase (Fig. 5) while the $\delta$ functions yield several windows [see case (b) in Sec. II]. For $\omega=0.1$ (not shown) a two-step staircase is seen for $L=4$, while two and one windows are found for $L=6$ and 8 , respectively.

\section{SUMMARY AND CONCLUSIONS}

We have studied how ratchet transport in inhomogeneous sine-Gordon systems can be strongly enhanced, proceeding in three steps.

(1) In a collective coordinate approach the soliton dynamics was represented by a relativistic particle ratchet with an effective potential $U$, which is periodic and asymmetric. Us- ing an ansatz in terms of Jacobi elliptic functions, the optimal $U$ for the particle ratchet was found. $U_{\text {opt }}$ exhibits essentially two features: (a) double peaks consisting of a positive and a negative peak with a steep slope in between and (b) long flat parts between the double peaks.

(2) The inhomogeneities in the sine-Gordon system were chosen such that they produce an effective potential which exhibits the above features of $U_{\text {opt }}$. It turned out that a very good choice is a periodic array in which each cell contains two very narrow and high boxes with opposite signs that touch each other (this structure can be well approximated by a $\delta^{\prime}$-function). The optimization of the parameters (width and height of the boxes and the cell length) yielded high values for $v_{\max }$, the maximum of the average soliton velocity $\langle v\rangle$ as a function of the driving amplitude $A$. For the frequency range $[0.015,0.1] v_{\max }$ is in the order of 0.15 , where the soliton speed $|v(t)|$ reaches values that are not far away from the critical velocity $c=1$. For frequencies higher than $\omega=0.2$ the ratchet effect vanishes.

(3) Simulations for the sine-Gordon system with the above inhomogeneities produce results for $\langle v\rangle$ vs $A$ which show a very good qualitative agreement with the results of the collective coordinate approach.

From the results that we have obtained, we propose the following experiments on long Josephson junctions: Narrow inhomogeneities of opposite signs can be built by microresistors (critical Josephson current $J_{c}$ decreased) and microshorts $\left(J_{c}\right.$ increased $)$. So far only equidistant arrays of either type of inhomogeneities were used, but we do not see problems in producing arrays in which microresistors are placed close to microshorts.

A further enhancement of $v_{\max }$ could be achieved by a replacement of the sinusoidal driving force $f(t)$ by a more general periodic force (for homogeneous sine-Gordon systems an ansatz in terms of Jacobi elliptic functions [see below Eq. (25)] yielded $v_{\max }=0.16$ and 0.17 in simulations and a CC approach, respectively [37]). Work on the joint optimization of the inhomogeneities and the driving force is in progress.

\section{ACKNOWLEDGMENTS}

F.G.M. acknowledges the hospitality of the Theoretical Division and Center for Nonlinear Studies at Los Alamos National Laboratory during his sabbatical stay. Work at Los Alamos is supported by the USDOE. Travel between Spain and Germany was supported by the Ministerio de Educación y Ciencia (Spain) and DAAD (Germany) through "Acciones Integradas Hispano-Alemanas" Grants No. HA2004-0034 and No. D/04/3997. A.S. is supported by the Ministerio de Educación y Ciencia (Spain) under Grants No. FIS20041001 and No. NAN2004-9087-C03-03, and by Comunidad de Madrid (Spain) under Grants No. UC3M-FI-05-007 and No. SIMUMAT-CM. The authors also thank N. R. Quintero (Sevilla) for copies of her work before publication and fruitful discussions. 
[1] P. Hänggi and R. Bartussek, in Nonlinear Physics of Complex Systems-Current Status and Future Trends, edited by J. Parisi, S. C. Müller, and W. Zimmermann, Lecture Notes in Physics Vol. 476 (Springer, Berlin, 1996).

[2] R. D. Astumian and P. Hänggi, Phys. Today 55(11), 33 (2002).

[3] F. Jülicher, A. Ajdari, and J. Prost, Rev. Mod. Phys. 69, 1269 (1997).

[4] P. Reimann, Phys. Rep. 361, 57 (2002).

[5] Ratchets and Brownian Motors: Basics, Experiments and Applications, edited by H. Linke [special issue of Appl. Phys. A: Mater. Sci. Process. 75, 2 (2002)].

[6] I. Zapata, R. Bartussek, F. Sols, and P. Hänggi, Phys. Rev. Lett. 77, 2292 (1996).

[7] F. Marchesoni, Phys. Rev. Lett. 77, 2364 (1996).

[8] F. Falo, P. J. Martínez, J. J. Mazo, and S. Cilla, Europhys. Lett. 45, 700 (1999).

[9] E. Trías, J. J. Mazo, F. Falo, and T. P. Orlando, Phys. Rev. E 61, 2257 (2000).

[10] M. Salerno and N. R. Quintero, Phys. Rev. E 65, 025602(R) (2002).

[11] G. Costantini, F. Marchesoni, and M. Borromeo, Phys. Rev. E 65, 051103 (2002).

[12] A. V. Gorbach, S. Denisov, and S. Flach, Chaos 16, 023125 (2006).

[13] S. Flach, Y. Zolotaryuk, A. E. Miroshnichenko, and M. V. Fistul, Phys. Rev. Lett. 88, 184101 (2002).

[14] M. Salerno and Y. Zolotaryuk, Phys. Rev. E 65, 056603 (2002).

[15] L. Morales-Molina, N. R. Quintero, F. G. Mertens, and A. Sánchez, Phys. Rev. Lett. 91, 234102 (2003).

[16] L. Morales-Molina, N. R. Quintero, A. Sánchez, and F. G. Mertens, Chaos 16, 013117 (2006).

[17] A. V. Ustinov, C. Coqui, A. Kemp, Y. Zolotaryuk, and M. Salerno, Phys. Rev. Lett. 93, 087001 (2004).

[18] R. Chacon and N. R. Quintero, e-print physics/0503125.

[19] L. Morales-Molina, F. G. Mertens, and A. Sánchez, Eur. Phys.
J. B 37, 79 (2004).

[20] L. Morales-Molina, F. G. Mertens, and A. Sánchez, Phys. Rev. E 72, 016612 (2005).

[21] A. V. Ustinov, Phys. Lett. A 136, 155 (1989).

[22] M. V. Fistul, Sov. Phys. JETP 69, 209 (1989).

[23] M. Weides, M. Kemmler, E. Goldobin, H. Kohlstedt, R. Waser, D. Koelle, and R. Kleiner, e-print cond-mat/0605656.

[24] R. Gross, in Interfaces in High- $T_{c}$ Superconducting Systems, edited by S. L. Shinde and D. A. Rudman (Springer, New York, 1994), pp. 176-210.

[25] M. Remoissenet, Waves Called Solitons (Springer, Berlin, 1999).

[26] J. M. R. Parrondo and B. J. Cisneros, Appl. Phys. A: Mater. Sci. Process. 75, 179 (2002).

[27] A. V. Ustinov, Appl. Phys. Lett. 80, 3153 (2002).

[28] M. Beck, E. Goldobin, M. Neuhaus, M. Siegel, R. Kleiner, and D. Koelle, Phys. Rev. Lett. 95, 090603 (2005).

[29] M. J. Rice and E. J. Mele, Solid State Commun. 35, 487 (1980); M. J. Rice, Phys. Rev. B 28, 3587 (1983).

[30] N. R. Quintero, A. Sánchez, and F. G. Mertens, Phys. Rev. Lett. 84, 871 (2000).

[31] N. R. Quintero, A. Sánchez, and F. G. Mertens, Phys. Rev. E 62, 5695 (2000).

[32] MATHEMATICA 5.0 (Wolfram Research Inc., Champaign, IL, 2003).

[33] B. Lindner and L. Schimansky-Geier, Phys. Rev. Lett. 89, 230602 (2002).

[34] M. Schreier, P. Reimann, P. Hänggi, and E. Pollak, Europhys. Lett. 44, 416 (1998).

[35] M. San Miguel and R. Toral, in Instabitities and Nonequilibrium structures VI, edited by E. Tirapegui, J. Martínez, and R. Tieman (Kluwer Academic, Dordrecht, 2000), pp. 35-129.

[36] W. A. Strauss and L. Vázquez, J. Comput. Phys. 28, 271 (1978).

[37] R. Chacon and N. R. Quintero, in Proceedings of BIOCOMP 2005 (unpublished) 